\title{
Linking information reconciliation and privacy amplification
}

\section{Journal Article}

Author(s):

Cachin, Christian; Maurer, Ueli M.

Publication date:

1997

Permanent link:

https://doi.org/10.3929/ethz-b-000422796

Rights / license:

In Copyright - Non-Commercial Use Permitted

Originally published in:

Journal of Cryptology 10(2), https://doi.org/10.1007/s001459900023 


\title{
Linking Information Reconciliation and Privacy Amplification*
}

\author{
Christian Cachin and Ueli M. Maurer \\ Institute for Theoretical Computer Science, ETH Zürich, \\ $\mathrm{CH}-8092$ Zürich, Switzerland \\ cachin@acm.org; maurer@inf.ethz.ch \\ Communicated by Gilles Brassard
}

Received 14 June 1994 and revised 30 October 1995

\begin{abstract}
Information reconciliation allows two parties knowing correlated random variables, such as a noisy version of the partner's random bit string. to agree on a shared string. Privacy amplification allows two parties sharing a partially secret string about which an opponent has some partial information, to distill a shorter but almost completely secret key by communicating only over an insecure channel, as long as an upper bound on the opponent's knowledge about the string is known. The relation between these two techniques has not been well understood. In particular, it is important to understand the effect of side-information. obtained by the opponent through an initial reconciliation step, on the size of the secret key that can be distilled safely by subsequent privacy amplification. The purpose of this paper is to provide the missing link between these techniques by presenting bounds on the reduction of the Rényi entropy of a random variable induced by side-information. We show that, except with negligible probability, each bit of side-information reduces the size of the key that can be safely distilled by at most two bits. Moreover, in the important special case of side-information and raw key data generated by many independent repetitions of a random experiment, each bit of side-information reduces the size of the secret key by only about one bit. The results have applications in unconditionally secure key agreement protocols and in quantum cryptography.
\end{abstract}

Key words. Unconditional security, Reconciliation. Information reduction. Privacy anplification, Rényi entropy, Secret-key agreement, Quantum cryptography.

\section{Introduction}

One of the fundamental problems in cryptography is the generation of a shared secret key by two parties, Alice and Bob, not sharing a secret key initially, in the presence of an adversary Eve. One generally assumes that Eve can eavesdrop on the communication

\footnotetext{
* This research was supported by the Swiss National Science Foundation. A preliminary version of this paper was presented at Eurocrypt '94. May 9-12. Perugia, Italy.
} 
between Alice and Bob who are connected only by a public channel. It is easy to see that if this public channel is not assumed to be authenticated, then such key agreement is impossible. We therefore assume that any modification or insertion of messages can be detected by Alice and Bob.

This problem can be solved by applying public-key cryptography [9], where one assumes that Eve's computing power is limited and that certain problems are computationally difficult. In the recent years, key agreement protocols have been developed that are secure against adversaries with unlimited computing power [1], [10]. The motivation for investigating such protocols is twofold. First, one avoids having to worry about the generality of a particular computational model, which is of some concern in view of the potential realizability of quantum computers [5], [13]. Second, no strong rigorous results on the difficulty of breaking a cryptosystem have been proved, and this problem continues to be among the most difficult ones in complexity theory.

Unconditionally secure secret-key agreement [10], [11] takes place in a scenario where Alice, Bob, and Eve know the correlated random variables $X, Y$, and $Z$, respectively, distributed according to some joint probability distribution that may be under the partial control of Eve (as, for instance, in quantum cryptography [1]). One possible scenario considered by Maurer [10] is that $X, Y$, and $Z$ result from a binary random string broadcast by a satellite and received by Alice, Bob, and Eve over noisy channels. Secret-key agreement is possible even when Eve's channel is much more reliable than Alice's and Bob's channels.

A key agreement protocol for such a scenario generally consists of three phases:

Advantage Distillation [10]. The purpose of the first phase is to create a random variable $W$ about which both Alice or Bob have more information than Eve. Advantage distillation is only needed when such a $W$ is not immediately available from $X$ and $Y$, for instance, when Eve's channel is superior in the above satellite scenario. Alice and Bob create $W$ by exchanging messages, summarized as the random variable $C$, over the public channel.

Information Reconciliation [1], [6]. To agree on a string $T$ with very high probability, Alice and Bob exchange redundant error-correction information $U$, such as a sequence of parity checks. After this phase, Eve's (incomplete) information about $T$ consists of $Z, C$, and $U$.

Privacy Amplification [2], [3]. In the final phase, Alice and Bob agree publicly on a compression function $G$ to distill from $T$ a shorter string $S$ about which Eve has only a negligible amount of information. Therefore, $S$ can subsequently be used as a secret key.

Information reconciliation and privacy amplification are fundamental for unconditionally secure key agreement and quantum key distribution. Advantage distillation has not yet been used in quantum cryptography because the considered scenarios assume that Alice and Bob have an advantage compared to Eve.

If after the first phase Alice knows a string about which Bob has more information than Eve, Alice and Bob can choose $W$ to be this string. In other words, using informationtheoretic terms, $W$ is a random variable such that $H(W \mid X C)=0$ and $H(W \mid Y C)<$ $H(W \mid Z C)$. In such a case, Bob tries to determine $W$ from $Y$ and the reconciliation string 
$U$. (Note that $H(U) \geq H(W \mid Y C)$ is a necessary condition.) Hence reconciliation serves to establish $H(W \mid Y C U) \approx 0$ while Eve still has a substantial amount of uncertainty about $W: H(W \mid Z C U)>0$. After privacy amplification, $H(S)$ should be as large as possible, and Eve's information about $S$ should be arbitrarily close to zero: $I(S ; Z C U G)=H(S)$ $H(S \mid Z C U G) \approx 0$. Note that Alice and Bob can both compute $S$, i.e., $H(S \mid W G)=0$.

In the following, let $V=[Z, C]$ summarize Eve's total knowledge about $W$ before reconciliation. For deriving lower bounds on Eve's final information about the secret key $S$ one can either consider a particular value $V=v$ that Eve knows or one can average over all possible values of $V$. Note that results for a particular $V=v$, which will be considered in this paper, are stronger than averaging results because they are known to hold for the very instance of the protocol execution. In other words, Eve's information about $W$ is modeled by the probability distribution $P_{W \mid V=v}$ about which Alice and Bob have some incomplete knowledge. In particular, they know a lower bound on the Rényi entropy (see below) of the distribution $P_{W \mid V=v}$ with high probability but they do not know $v$.

It is known [2] that the Rényi entropy after reconciliation with $U=u$ (i.e., of the distribution $P_{W \mid V=v, U=u}$ ) is a lower bound on the size of the secret key that can be distilled safely by privacy amplification. This paper is concerned with understanding the reduction of the Rényi entropy induced by the side information $U$, either for a particular value $U=u$, or averaged over all values of $U$. Although this question is fundamental for any proof in the area of key agreement protocols, it has previously not been well understood because the behavior of Renyi entropy is different from that of Shannon entropy with respect to side-information. Existing proofs such as the ingenious Big Brother argument of [1] work only for particular probability distributions and reconciliation protocols.

The paper is organized as follows. Section 2 reviews privacy amplification and the definition of Rényi entropy. Section 3 presents upper bounds on the reduction of Rényi entropy due to side-information for arbitrary probability distributions. Noninteractive reconciliation protocols with uniform and close-to-uniform probability distributions are investigated in Section 4. These results are applied in Section 5 to analyze the important class of scenarios in which a given random experiment is repeated many times independently.

\section{Review of Privacy Amplification and Rényi Entropy}

We assume that the reader is familiar with the notion of entropy and the basic concepts of Shannon's information theory [4], [8]. In privacy amplification, a different entropy measure, Rényi entropy, is of central importance [2]. To distinguish Rényi entropy from entropy in the sense of Shannon, we will always refer to the latter as Shannon entropy. All logarithms in this paper are to the base 2, and entropies are thus measured in bits.

Privacy amplification was introduced by Bennett $e t$ al. [3] and investigated further in [2], and can be described as follows. Assume Alice and Bob share an $n$-bit string $W$ about which an eavesdropper Eve has incomplete information characterized by a probability distribution $P_{W \mid V=v}$ over the $n$-bit strings, where $v$ denotes the particular value taken on by the random variable $V$ summarizing her side-information. For instance, Eve might have received some bits or parities of bits of $W$, she might have eavesdropped 
on some of the bits of $W$ through a binary symmetric channel, or have some more complicated type of information about $W$. Alice and Bob have some knowledge of the distribution $P_{W \mid V=v}$, but they do not know exactly what is compromised about their string. Using a public channel, which is totally susceptible to eavesdropping but immune to tampering, they wish to agree on a function $g:\{0,1\}^{n} \rightarrow\{0,1\}^{r}$ such that Eve, despite her partial knowledge about $W$ and complete knowledge of $g$, almost certainly knows nearly nothing about $g(W)$. This process transforms a partially secret $n$-bit string $W$ into a highly secret but shorter $r$-bit string $g(W)$ which can be used as a secret key.

The method for selecting the function $g$ proposed in [3] is to choose it at random from a publicly known universal class of hash functions mapping $n$-bit strings to $r$-bit strings. Universal hash functions were introduced by Carter and Wegman [7]. A class $G$ of functions $\mathcal{A} \rightarrow \mathcal{B}$ is called universal if, for any distinct $x_{1}$ and $x_{2}$ in $\mathcal{A}$, the probability that $g\left(x_{1}\right)=g\left(x_{2}\right)$ is at most $1 /|\mathcal{B}|$, when $g$ is chosen at random with uniform distribution from $G$.

Bennett et al. [2] showed that the Rényi entropy (defined below) of Eve's distribution about $W$ provides a lower bound on the size $r$ of the secret key distillable from $W$ by privacy amplification with a universal hash function.

Definition 1. Let $X$ be a random variable with alphabet $\mathcal{X}$ and distribution $P_{X}$. The collision probability $P_{c}(X)$ of $X$ is defined as the probability that $X$ takes on the same value twice in two independent experiments:

$$
P_{c}(X)=\sum_{x \in X} P_{X}(x)^{2} .
$$

The Rényi entropy of order two (or "Rényi entropy" for short) of $X$ [12], [2] is defined as the negative logarithm of the collision probability of $X$ :

$$
R(X)=-\log P_{c}(X) .
$$

For an event $\mathcal{E}$, the Rényi entropy of $X$ conditioned on $\mathcal{E}, R(X \mid \mathcal{E})$, is defined naturally as the Rényi entropy of the conditional distribution $P_{X \mid \mathcal{E}}$. The Rényi entropy conditioned on a random variable, $R(X \mid Y)$, is defined as the expected value of the conditional Rényi entropy:

$$
R(X \mid Y)=\sum_{y} P_{Y}(y) R(X \mid Y=y)
$$

Equivalently, $R(X)$ can be expressed as $R(X)=-\log E\left[P_{X}(X)\right]$, where $E[\cdot]$ denotes the expected value. Shannon entropy $H(X)$ can be expressed similarly as $H(X)=$ $-E\left[\log P_{X}(X)\right]$. It follows from Jensen's inequality (sce [8]) that Rényi entropy is upper bounded by the Shannon entropy, a fact known to Rényi:

$$
R(X) \leq H(X)
$$

with equality if and only if $P_{X}$ is the uniform distribution over $\mathcal{X}$ or a subset of $\mathcal{X}$. Similarly, we have $H(X \mid Y) \geq R(X \mid Y)$. Note that Rényi entropy (like Shannon entropy) is always positive.

The following theorem is the main result of [2]. 
Theorem 1. Let $X$ be a random variable on alphabet $\mathcal{X}$ with probability distribution $P_{X}$ and Renyi entropy $R(X)$. Further, let $G$ be the random variable corresponding to the random choice (with uniform distribution) of a member of a universal class of hash functions from $\mathcal{X} \rightarrow\{0,1\}^{r}$. Then

$$
H(G(X) \mid G) \geq R(G(X) \mid G) \geq r-\frac{2^{r-R(X)}}{\ln 2} .
$$

Note that $G$ is a random variable and that the quantity $H(G(X) \mid G)$ is an average over all choices of the function $g$. It is possible that $H(G(X) \mid G=g)=H(g(X))$ differs from $r$ by a nonnegligible amount for some $g$, but such a $g$ can occur only with negligible probability.

This theorem clearly applies also to conditional probability distributions such as $P_{W \mid V=v}$ discussed above. If Eve's Rényi entropy $R(W \mid V=v)$ is known to be at least $t$ and Alice and Bob choose $S=G(W)$ as their secret key, then

$$
R(S \mid G, V=v)=R(G(W) \mid G, V=v) \geq r-\frac{2^{r-l}}{\ln 2} .
$$

The key $S$ is indeed virtually secret because $H(S \mid G, V=v) \geq R(S \mid G . V=v)$ and hence $H(S \mid G, V=v)$ is arbitrarily close to maximal. More precisely, if $r<t$, then Eve's total information about $S$ decreases exponentially in the excess compression $t-r$.

It should be pointed out that Theorem 1 cannot be generalized to Rényi entropy conditioned on a random variable, i.e., $R(G(W) \mid G V) \geq r-2^{r-R(W \mid V)} / \ln 2$ is false in general [2].

\section{The Effect of Side Information on Rényi Entropy}

As described above, the reconciliation step consists of Alice and Bob exchanging suitable error-correction information $U$ over the public channel. This information decreases Eve's Shannon entropy and usually also her Rényi entropy about $W$. For noninteractive reconciliation, Alice chooses an appropriate error-correction function $f$ and sends $U=f(W)$ to Bob who can then reconstruct $W$ with high probability from $U$ and his prior knowledge $Y C$.

The results of this paper will be derived for an arbitrary random variable $X$ with probability distribution $P_{X}$ and a side-information random variable $U$ jointly distributed with $X$ according to $P_{X U}$. However, they can just as well be applied to conditional distributions; our intended application is the key agreement scenario mentioned in the introduction, i.e., when $P_{X}$ and $P_{X \mid U}$ are replaced by $P_{W \cdot V=v}$ and $P_{W \mid V=r, U}$, respectively.

In general, giving side-information implies a reduction of entropy. Our goal is to derive upper bounds on the size of this reduction. Giving as side-information the fact that $U$ takes on a particular value $u$, it is possible for both, Shannon and Rényi entropies, that the entropy increases or decreases. Moreover, the size of a reduction can be arbitrarily large. However, the expected reduction (for all values of $U$ ) of the Shannon entropy of $X$ by giving $U$, called the mutual information between $X$ and $U$, is bounded by $H(U)$ :

$$
H(X)-H(X \mid U)=I(X ; U) \leq H(U),
$$


which follows from the symmetry of $I(X ; U)$ and the fact that Shannon entropy (conditional or not) is always positive.

The example below illustrates two facts. First, the reduction of Rényi entropy implied by giving side-information $U=u$ can exceed the reduction of Shannon entropy, i.e.,

$$
R(X)-R(X \mid U=u)>H(X)-H(X \mid U=u)
$$

is possible. Second, it shows that the natural generalization of (1) to Rényi entropy, namely $R(X)-R(X \mid U) \leq R(U)$, is not true in general. However, Theorem 2 demonstrates that the weaker inequality $R(X)-R(X \mid U) \leq H(U)$ is always satisfied.

Example. Let $X$ be a random variable with alphabet $\mathcal{X}=\left\{a_{1}, \ldots, a_{10}, b_{1}, \ldots, b_{10}\right\}$, distributed according to $P_{X}\left(a_{i}\right)=0.01$ and $P_{X}\left(b_{i}\right)=0.09$ for $i=1, \ldots, 10$. We have $H(X) \approx 3.79$ and $R(X) \approx 3.61$. Let $f: \mathcal{X} \rightarrow\{0,1\}$ be defined as

$$
f(x)= \begin{cases}0 & \text { if } \quad x \in\left\{a_{1}, \ldots, a_{9}, b_{10}\right\}, \\ 1 & \text { if } \quad x \in\left\{a_{10}, b_{1}, \ldots, b_{9}\right\},\end{cases}
$$

and let $U=f(X)$. Then $H(X \mid U=0) \approx 2.58$ and $R(X \mid U=0) \approx 1.85$. The reduction of Rényi entropy when given $U=0$ exceeds the reduction of Shannon entropy, i.e., $R(X)-R(X \mid U=0) \approx 1.76$ whereas $H(X)-H(X \mid U=0) \approx 1.21$.

Because because $f$ is deterministic, $H(U)=H(X)-H(X \mid U) \approx 0.69$. The expected entropy reductions are $H(X)-H(X \mid U) \approx 0.69$ and $R(X)-R(X \mid U) \approx 0.65$. Note that $R(U) \approx 0.50$ and that $R(X)-R(X \mid U)$ is indeed greater than $R(U)$ but less than $H(U)$.

$H(U)$ is not only the maximal expected decrease of Shannon entropy, but $H(U)$ is also an upper bound on the expected decrease of Rényi entropy, as the following theorem demonstrates.

Theorem 2. Let $X$ and $U$ be two random variables with alphabets $\mathcal{X}$ and $\mathcal{U}$, respectively. The expected reduction of the Rényi entropy of $X$, when given $U$, does not exceed the Shannon entropy of $U$, i.e..

$$
R(X)-R(X \mid U) \leq H(U),
$$

with equality if and only if $U$ is defined uniquely for each $x \in \mathcal{X}$ and $P_{U}$ is the uniform distribution over $\mathcal{U}$ or a subset of $\mathcal{U}$.

Proof. The collision probability of $X$ can be written as

$$
\begin{aligned}
P_{c}(X) & =\sum_{x \in \mathcal{X}} P_{X}(x)^{2} \\
& =\sum_{x \in \mathcal{X}}\left(\sum_{u \in \mathcal{U}} P_{X U}(x, u)\right)^{2} \\
& \geq \sum_{x \in \mathcal{X}} \sum_{u \in \mathcal{U}} P_{X U}(x, u)^{2}
\end{aligned}
$$




$$
\begin{aligned}
& =\sum_{u \in \mathcal{L} u} P_{U}(u)^{2} \sum_{x \in \mathcal{X}^{\prime}} P_{X \mid U}(x, u)^{2} \\
& =\sum_{u \in \mathcal{U}} P_{U}(u)^{2} P_{c}(X \mid U=u),
\end{aligned}
$$

where the inequality follows from $\left(\sum_{i=1}^{n} p_{i}\right)^{2} \geq \sum_{i=1}^{n} p_{i}^{2}$ for nonnegative $p_{i}(1 \leq i \leq n)$ and equality holds if and only if $p_{i}=0$ for all but one $i$. Inserting (2) into the definition of Rényi entropy gives

$$
\begin{aligned}
R(X) & =-\log P_{c}(X) \\
& \leq-\log \left(\sum_{u \in \mathcal{U}} P_{U^{\prime}}(u)^{2} P_{c}(X \mid U=u)\right) \\
& =-\log \left(\sum_{u \in \mathcal{U}} P_{U}(u)\left[P_{U}(u) P_{c}(X \mid U=u)\right]\right) \\
& \leq-\sum_{u \in \mathcal{U}} P_{U}(u) \log \left[P_{U}(u) P_{u}(X \mid U=u)\right] \\
& =-\sum_{u \in \mathcal{U}} P_{U}(u)\left[\log P_{U}(u)+\log P_{u}(X \mid U=u)\right] \\
& =-\sum_{u \in \mathcal{U}} P_{U}(u) \log P_{U}(u)-\sum_{u \in \mathcal{U}} P_{U}(u) \log P_{c}(X \mid U=u) \\
& =H(U)+\sum_{u \in \mathcal{U}} P_{U}(u) R(X \mid U=u) \\
& =H(U)+R(X \mid U),
\end{aligned}
$$

where the second inequality follows from Jensen's inequality [8] which holds with equality if and only if $P_{U}$ is the uniform distribution over $\mathcal{U}$ or a subset of $\mathcal{U}$.

In contrast to Shannon entropy, the expected Rényi entropy can increase when side information is revealed, i.e., $R(X)<R(X \mid U)$ is possible. This property is used in [2| and [11] to prove that a key larger than suggested by Theorem 1 can be obtained by privacy amplification. The proof makes use of a conceptual oracle that is assumed to provide special side-information $U$ to Eve, called spoiling knowledge. which increases her Rényi entropy. Of course, this extra information cannot harm her because she could always discard it.

For example, if Eve has received $V=v$ over a binary symmetric channel, her Rényi entropy of the distribution $P_{W \mid V=v}$ is considerably lower than her Shannon entropy. But the oracle can increase her Rényi entropy with high probability almost to the Shannon entropy by telling her the number $d$ of bits in $v$ that she received incorrectly. Then, all strings at distance $d$ of $v$ are equally likely and Eve's Rényi entropy increases to the (new) Shannon entropy.

For a positive-valued random variable $X, E[X] \leq t$ implies that $P[X \geq k t] \leq 1 / k$. Hence, according to Theorem 2 , the probability that the leaking information $U=u$ decreases Rényi entropy by more than $k H(U)$ is at most $1 / k$, i.e.. $P[R(X)-R(X \mid U=$ $u) \geq k H(U)] \leq 1 / k$. However, such a high probability of partially exposing the string 
$W$ is unacceptable in a key agreement scenario. The following theorem provides a much stronger result by showing that the above probability decreases in fact at most by $2 \log |\mathcal{U}|$ except with negligible probability.

Theorem 3. Let $X$ and $U$ be random variables with alphabets $\mathcal{X}$ and $\mathcal{X}$, respectively, and let $s>0$ be an arbitrary security parameter. With probability at least $1-2^{-s}, U$ takes on a value $u$ for which

$$
R(X)-R(X \mid U=u) \leq 2 \log |\mathcal{U}|+2 s
$$

Remark. The statement of the theorem is equivalent to

$$
\sum_{u: R(X)-R(X \mid U=u) \leq 2 \log \mid \mathcal{U}+2 s} P_{U}(u) \geq 1-2^{-s} .
$$

Proof. We can bound the reduction only for those values $u$ for which $P_{U}(u)$ is not too small. Let $\mathcal{E}$ be the event that $U \in\left\{u \mid P_{U}(u) \geq p_{\min }\right\}$ for some given $p_{\min }>0$ and $p_{\min }<1 /|\mathcal{U}|$. There are $|\mathcal{U}|$ possible values $u$, and for at most $|\mathcal{U}|-1$ values $u$, $P_{U}(u)<p_{\min }$ can hold. Therefore we have $P[\overline{\mathcal{E}}] \leq|\mathcal{U}| \cdot p_{\min }$ and $P\left[\mathcal{E}|\geq 1-| \mathcal{U} \mid \cdot p_{\min }\right.$. If the event $\mathcal{E}$ occurs we have

$$
\begin{aligned}
P_{c}(X \mid U=u) & =\sum_{x \in \mathcal{X}} P_{X, U}(x, u)^{2} \\
& =\sum_{x \in \mathcal{X}^{\prime}}\left(\frac{P_{X U}(x, u)}{P_{U}(u)}\right)^{2} \\
& \leq \sum_{x \in \mathcal{X}^{\prime}} \frac{P_{X}(x)^{2}}{P_{U}(u)^{2}} \\
& \leq \sum_{x \in \mathcal{X}} \frac{P_{X}(x)^{2}}{P_{\min }^{2}} \\
& =\frac{P_{c}(X)}{p_{\min }^{2}}
\end{aligned}
$$

where we have made use of the fact that $P_{X U}(x, u)=P_{X}(x) P_{U \mid X}(u, x) \leq P_{X}(x)$. If we set $p_{\min }=2^{-s} /|\mathcal{U}|$, then $P_{U}(u)>p_{\min }$ with probability $P[\mathcal{E}] \geq 1-2^{-s}$. The theorem now follows by taking logarithms on both sides of

$$
P_{i}(X \mid U=u) \leq|\mathcal{U}|^{2} 2^{2 s} P_{c}(X) .
$$

Because of its importance we restate Theorem 3 for the key-generation scenario, replacing $P_{X}$ by $P_{W \mid V=v}$, with the side-information consisting of $k$ bits, for instance $k$ parity checks of $W$ when $W$ is an $n$-bit string.

Corollary 4. Let $W$ be a random variable with alphabet $\mathcal{W}$, let $v$ and $u$ be particular values of the correlated random variables $V$ and $U$ with alphabets $\mathcal{V}$ and $\mathcal{U}$, respectively, 
with $k=\log |\mathcal{U}|$, and let $s>0$ be a given security parameter. Then, with probability at least $1-2^{-s}, U$ takes on a value u such that the decrease in Rényi entropy by giving $u$,

$$
R(W \mid V=v)-R(W \mid V=v, U=u) .
$$

is at most $2 k+2 s$.

\section{Almost Uniform Distributions}

As shown above, giving side-information of the form $U=u$ can reduce the Rényi entropy by an arbitrary amount, although the probability that this happens is bounded by Theorem 3. In this section and the next we derive better bounds on the reduction for noninteractive reconciliation and special probability distributions. For uniform distributions and deterministic side-information $U=f(W)$ the reduction of Rényi entropy depends only on the size of the preimage of $u=f(x)$.

Lemma 5. Let $X$ be a random variable with alphabet $\mathcal{X}$, let $f: \mathcal{X} \rightarrow \mathcal{U}$ be an arbitrary function taking on values in a given set $\mathcal{U}$, let $U$ be defined as $U=f(X)$, and set $\mathcal{X}_{u}=\{x \in \mathcal{X}: f(x)=u\}$. If $X$ is distributed uniformly over $\mathcal{X}$, then

$$
R(X)-R(X \mid U=u)=\log \frac{|\mathcal{X}|}{\left|\mathcal{X}_{u}\right|} .
$$

In particular, if $f$ is such that $\left|\mathcal{X}_{u}\right|$ is the same for all $u \in \mathcal{U}$, knowledge of $U=u$ reduces the Rénvi entropy by $\log |\mathcal{U}|$.

Proof. Because Rényi entropy equals Shannon entropy for the uniform distribution, we have $R(X)=\log |\mathcal{X}|$ and $R(X \mid U=u)=\log \left|\mathcal{X}_{u}\right|$. from which the first claim immediately follows. To prove the second claim, note that in this case $|\mathcal{X}| /\left|\mathcal{X}_{u}\right|=|\mathcal{U}|$ for all $u \in \mathcal{U}$.

Theorems 6 and 7 state bounds on the reduction of Rényi entropy for almost uniform distributions. These results are applied in the next section to the analysis of the important class of scenarios where a given random experiment is repeated many times independently.

Theorem 6. For given $\alpha>1$ and $\beta>1$, let $X$ be a random variable with alphabet $\mathcal{X}$ and probability distribution $P_{X}$ such that $1 /(\alpha|\mathcal{X}|) \leq P_{X}(x) \leq \beta /|\mathcal{X}|$ for all $x \in \mathcal{X}$. Define $f, U$, and $\mathcal{X}_{u}$ as in Lemma 5. Then

$$
R(X)-R(X \mid U=u) \leq \log \frac{|\mathcal{X}|}{\left|\mathcal{X}_{u}\right|}+4 \log \alpha+2 \log \beta .
$$

In particular, if $f$ is such that $\left|\mathcal{X}_{u}\right|$ is the same for all $u \in \mathcal{U}$, then $R(X)-R(X \mid U=$ u) $\leq \log |\mathcal{U}|+4 \log \alpha+2 \log \beta$. 
Proof. We can bound $P_{c^{\prime}}(X)$ as follows:

$$
P_{c}(X)=\sum_{x \in \mathcal{X}^{\prime}} P_{X}(x)^{2} \geq|\mathcal{X}| \frac{1}{(\alpha|\mathcal{X}|)^{2}}=\frac{1}{\alpha^{2}|\mathcal{X}|}
$$

Using $P_{U}(u) \geq\left|\mathcal{X}_{u}\right| /(\alpha|\mathcal{X}|)$ we get a similar upper bound for $P_{c}(X \mid U=u)$ :

$$
\begin{aligned}
P_{c}(X \mid U=u) & =\sum_{x \in \mathcal{X}_{u}} P_{X ! U}(x, u)^{2} \\
& =\frac{1}{P_{U}(u)^{2}} \sum_{x \in \mathcal{X}_{u}^{\prime}} P_{X}(x)^{2} \\
& \leq\left(\frac{\alpha|\mathcal{X}|}{\left|\mathcal{X}_{u}\right|}\right)^{2}\left|\mathcal{X}_{u}\right|\left(\frac{\beta}{|\mathcal{X}|}\right)^{2}=\frac{\alpha^{2} \beta^{2}}{\left|\mathcal{X}_{u}\right|} .
\end{aligned}
$$

Combining (3) and (4) gives

$$
\frac{P_{c}(X \mid U=u)}{P_{c}(X)} \leq \frac{|\mathcal{X}|}{\left|\mathcal{X}_{u}\right|} \alpha^{4} \beta^{2}
$$

and the theorem follows by taking logarithms on both sides.

The following theorem provides a tighter bound for distributions that are very close to uniform. In particular, Theorem 7 is strictly tighter than Theorem 6 for $\gamma \leq 0.4563$. For $0 \leq \gamma \leq 0.3$ it is about $30 \%$ tighter.

Theorem 7. For given $\gamma<\frac{1}{2}$, let $X$ be a random variable with alphabet $\mathcal{X}$ and probability distribution $P_{X}$ such that $(1-\gamma) /|\mathcal{X}| \leq P_{X}(x) \leq(1+\gamma) /|\mathcal{X}|$ for all $x \in \mathcal{X}$. Define $f . U$, and $\mathcal{X}_{u}$ as in Lemma 5. Then

$$
R(X)-R(X \mid U=u) \leq \log \frac{|\mathcal{X}|}{\left|\mathcal{X}_{u}\right|}+\log \frac{(1+\gamma)^{2}}{1-2 \gamma}
$$

Proof. For each $x$, define $\delta_{x}$ as the deviation of $P_{X}(x)$ from the uniform distribution: $P_{X}(x)=1 /|\mathcal{X}|+\delta_{x}$. Hence we have $\left|\delta_{x}\right| \leq \gamma /|\mathcal{X}|$, and $P_{c}(X \mid U=u)$ can be expressed as follows:

$$
\begin{aligned}
P_{c}(X \mid U=u) & =\sum_{x \in \mathcal{X}_{u}} P_{X \mid U}(x, u)^{2} \\
& =\sum_{x \in \mathcal{X}_{u}^{\prime}}\left(\frac{P_{X U}(x, u)}{P_{U}(u)}\right)^{2} \\
& =\frac{\sum_{x \in \mathcal{X}_{u}} P_{X}(x)^{2}}{P_{U}(u)^{2}} \\
& =\frac{\sum_{x \in \mathcal{X}_{u}} P_{X}(x)^{2}}{\left(\sum_{x \in \mathcal{X}_{u}} P_{X}(x)\right)^{2}}
\end{aligned}
$$




$$
\begin{aligned}
& =\frac{\sum_{x \in \mathcal{X}_{u}}\left(1 /|\mathcal{X}|+\delta_{x}\right)^{2}}{\left(\sum_{x \in \mathcal{X}_{u}} 1 /|\mathcal{X}|+\sum_{x \in \mathcal{X}_{u}} \delta_{x}\right)^{2}} \\
& =\frac{\left|\mathcal{X}_{u}\right| /|\mathcal{X}|^{2}+2 /|\mathcal{X}| \sum_{x \in \mathcal{X}_{u}} \delta_{x}+\sum_{x \in \mathcal{X}_{u}} \delta_{x}^{2}}{\left|\mathcal{X}_{u}\right|^{2} /|\mathcal{X}|^{2}+2\left|\mathcal{X}_{u}\right| /|\mathcal{X}| \sum_{x \in \mathcal{X}_{u}} \delta_{x}+\left(\sum_{x \in \mathcal{X}_{u}} \delta_{x}\right)^{2}} \\
& \leq \frac{\left|\mathcal{X}_{u}\right| /|\mathcal{X}|^{2}+(2 /|\mathcal{X}|)\left|\mathcal{X}_{u}\right|(\gamma /|\mathcal{X}|)+\left|\mathcal{X}_{u}\right|\left(\gamma^{2} /|\mathcal{X}|^{2}\right)}{\left|\mathcal{X}_{u}\right|^{2} /|\mathcal{X}|^{2}-2\left(\left|\mathcal{X}_{u}\right| /|\mathcal{X}|\right)\left|\mathcal{X}_{u}\right|(\gamma /|\mathcal{X}|)} \\
& =\frac{1+2 \gamma+\gamma^{2}}{\left|\mathcal{X}_{u}\right|-2 \gamma\left|\mathcal{X}_{u}\right|}
\end{aligned}
$$

In the third step we have made use of the fact that $U$ is a deterministic function of $X$ and thus $P_{X U}(x, u)=P_{X}(x)$. Using $P_{c}(X) \geq 1 /|\mathcal{X}|$, we get

$$
\frac{P_{c}(X \mid U=u)}{P_{c}(X)} \leq \frac{|\mathcal{X}|}{\left|\mathcal{X}_{u}\right|} \cdot \frac{(1+\gamma)^{2}}{1-2 \gamma},
$$

from which the theorem follows.

\section{Independent Repetition of a Random Experiment}

In many practical scenarios, a certain random experiment is repeated independently a large number of times. For example, $W$ could be the result of receiving independently generated bits over a memoryless channel, as in the satellite scenario mentioned earlier. A fundamental theorem of information theory states that in such a scenario all occurring sequences can be divided into a typical set and a nontypical set, where the probability that a randomly selected sequence of Jength $n$ lies in the typical set approaches 1 for all sufficiently large $n$. Furthermore, all sequences in the typical set are almost equally probable. This allows us to bound the decrease of Rényi entropy by the results of the last section.

In the following we will make use of strongly typical sequences [4]. Consider a probability distribution $P_{X}$ over some finite set $\mathcal{X}$ where we assume $P_{X}(x)>0$ for all $x \in \mathcal{X}$. Let $x^{n}=\left[x_{1}, \ldots, x_{n}\right]$ be a sequence of $n$ digits of $\mathcal{X}$ and define $N_{a}\left(x^{n}\right)$ to be the number of occurrences of the symbol $a \in \mathcal{X}$ in the sequence $x^{n}$. A sequence $x^{n} \in \mathcal{X}^{n}$ is called $\varepsilon$-strongly typical if and only if

$$
(1-\varepsilon) P_{X}(a) \leq \frac{N_{a}\left(x^{n}\right)}{n} \leq(1+\varepsilon) P_{X}(a)
$$

for all $a \in \mathcal{X}$. Let $\mathcal{S}^{n}(\varepsilon)$ be the set of all $\varepsilon$-strongly typical sequences of length $n$ and define $X^{n}$ to be a sequence of $n$ independent and identically distributed random variables $X_{i}$ with $P_{X_{i}}=P_{X}$ for $1 \leq i \leq n$. In other words, we have $P_{X^{n}}\left(x^{n}\right)=\prod_{i=1}^{n} P_{X}\left(x_{i}\right)$. Let $O(n)$ be any function of $n$ such that $\lim _{n \rightarrow \infty} o(n)=0$. The following lemma asserts that, for sufficiently large $n$, the probability that $X^{n} \in \mathcal{S}^{n}(\varepsilon)$ approaches 1 and that the cardinality of $\mathcal{S}^{n}(\varepsilon)$ is close to $2^{n H(X)}$. 
Lemma 8 [4]. Let $X^{n}$ be a sequence of i.i.d. random variables distributed according to $P_{X}$. Then

1. For every $\delta>0, P\left[X^{n} \in \mathcal{S}^{n}(\varepsilon)\right] \geq 1-\delta / n$, for sufficiently large $n$.

2. For all $x^{n} \in \mathcal{S}^{n}(\varepsilon): P_{X^{n}}\left(x^{n}\right)=2^{-n} H(X)+o(n)$.

3. $\left|\mathcal{S}^{n}(\varepsilon)\right|=2^{n H(X)+o(n)}$.

Because all sequences in $\mathcal{S}^{n}(\varepsilon)$ are almost equally probable for sufficiently large $n$, the reduction of Rényi entropy is similar to the case of the uniform probability distribution where Rényi entropy behaves like Shannon entropy. This observation is stated as the next theorem.

Theorem 9. Let $X^{n}$ be a sequence of i.i.d. random variables distributed according to $P_{X}$, let $f: \mathcal{X}^{n} \rightarrow \mathcal{U}$ be an arbitrary function taking on values in a given set $\mathcal{U}$, and define $\mathcal{S}_{u}^{n}(\varepsilon)=\left\{x^{n} \in \mathcal{S}^{n}(\varepsilon): f\left(x^{n}\right)=u\right\}$ and $U=f\left(X^{n}\right)$. For any $\delta>0$ and sufficiently large $n$, the following holds with probability at least $1-\delta / n: X^{n}$ lies in $\mathcal{S}^{n}(\varepsilon)$ and the reduction of Rénvi entropy by giving $U=u$ is upper bounded by

$$
R\left(X^{n}\right)-R\left(X^{n} \mid U=u\right) \leq n H(X)-\log \left|\mathcal{S}_{u}^{n}(\varepsilon)\right|+o(n) .
$$

In particular, if $f$ is such that $|\{x \in \mathcal{X}: f(x)=u\}|$ is the same for all $u \in \mathcal{U}$ and $|\mathcal{U}|=2^{k}$, then knowledge of $U=u$ reduces the Rényi entropy by at most $k+o(n)$.

Proof. By Lemma 8, $P_{X^{n}}\left(x^{n}\right)=2^{-n H(X)+o(n)}$ for all $x^{n} \in \mathcal{S}^{n}(\varepsilon)$, and $\left|\mathcal{S}^{n}(\varepsilon)\right|=$ $2^{n H(X)+o(n)}$. Application of Theorem 6 with $\alpha=2^{o(n)}$ and $\beta=2^{o(n)}$ gives

$$
\begin{aligned}
R\left(X^{n}\right)-R\left(X^{n} \mid U=u\right) & \leq \log \frac{\left|\mathcal{S}^{n}(\varepsilon)\right|}{\left|\mathcal{S}_{u}^{n}(\varepsilon)\right|}+o(n) \\
& =n H(X)-\log \left|\mathcal{S}_{u}^{n}(\varepsilon)\right|+o(n)
\end{aligned}
$$

Note that the second part of the theorem applies in particular to all linear functions such as parity checks from linear error correcting codes. Due to their widespread use linear error correcting codes are most likely to be used during the reconciliation phase. Theorem 9 can replace the spoiling knowledge argument in Maurer's proof [11] that the known results on secret-key rate [10] hold also for a much stronger notion of secrecy.

\section{Conclusions}

The described link between information reconciliation and privacy amplification for unconditionally secure secret-key agreement can be summarized as follows. Assume that Alice knows a random variable $W$ and that Bob and Eve have partial knowledge about $W$, characterized by the random variables $W^{\prime}$ and $V$, respectively. These random variables could for instance result from the described satellite scenario with $W$ and $W^{\prime}$ being functions of $[X, C]$ and $[Y, C]$, respectively, and with $V=[Z, C]$. In order to state the results in the strongest possible form we consider a particular value $V=v$ held by Eve rather than the average over all values of $V$. 
When $V$ gives iess information than $W^{\prime}$ about $W$, i.e., $H(W \mid V)>H\left(W \mid W^{\prime}\right)$, and a lower bound $t>0$ on the Rényi entropy of Eve's probability distribution of $W$ is known, i.e., $R(W \mid V=v) \geq t$, then Alice and Bob can generate a shared secret key $S$ as follows. Alice and Bob exchange error-correcting information $U$ consisting of $k>H\left(W \mid W^{\prime}\right)$ bits over the public channel such that Bob can reconstruct $W$, i.e., $H\left(W \mid W^{\prime} U\right) \approx 0$. Eve gains additional knowledge about $W$ by seeing $U=u$. However, Corollary 4 shows that with probability at least $1-2^{-s}$ (over all values of $U$ ) where the security parameter $s$ can be chosen arbitrarily, her Rényi entropy is bounded from below by $R(W \mid V=v, U=u) \geq t-2 k-2 s$. Using privacy amplification, Alice and Bob can now generate an $r$-bit secret key $S$, where $r$ has to be chosen smaller than $t-2 k-2 s$ and Eve's total information about $S$ is exponentially small in $t-2 k-2 s-r$, namely

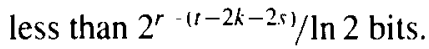

The main advantage of Theorem 3 is that it applies to any distribution and any reconciliation protocol whereas previously obtained results held only for particular distributions and protocols. However, as was demonstrated in Sections 4 and 5, a larger secret key than suggested by Theorem 3 can be obtained by Alice and Bob for speciai distributions. For instance, when Eve's information $V$ consists of a long sequence of independent random variables with identical distribution, then the term $2 k+2 s$ above can be replaced essentially by $k$ if noninteractive reconciliation is used. It is conceivable that the bound of Theorem 3 can be tightened for other special distributions than those treated in Sections 4 and 5 , and possibly even for general distributions.

\section{Acknowledgments}

It is a pleasure to thank Charles Bennett, Gilles Brassard, Claude Crépeau, and Martin Gander for interesting discussions and the anonymous referees for many helpful remarks.

\section{References}

[1] C. H. Bennett. F. Bessettc. (i. Brassard, L. Salvail, and J. Smolin. Experimental quantum cryptography. Joumal of Cryprology, vol. 5. no. 1, pp. 3-28. 1992.

[2] C. H. Bennett, C. Brassard, C. Crépeau, and U. M. Maurer, Generalized privacy amplification, IEEE Transactions on Information Theory, vol. 41. pp. 1915-1923, Nov. 1995.

[3] C. H. Bennett, G. Brassard, and J.-M. Robert, Privacy amplitication by public discussion, SIAM Journal on Computing. vol. 17. pp. 210-229. April 1988

[4] R. E. Blahut, Principles and Practice of Information Theory. Addison-Wesley. Reading, MA. 1987.

[5] G. Brassard, A quantum jump in computer science. In: Computer Science Toddoy (J. van Lecuwen, ed.), Lecture Notes in Computer Science, vol. 1000). pp. 1-14. Springer-Verlag. New York. 1995.

I6I G. Brassard and L. Salvail. Secret-key reconciliation by public discussion, In: Advances in CryptologyEUROCRYPT '93 (T. Helleseth. ed.). Lecture Notes in Computer Science. vol, 765, pp. 410-423, Springer-Verlag, New York, 1994.

17] J. L. Carter and M. N. Wegman, Universal classes of hash functions, Journal of Computer and System Sciences, vol. 18, pp. 143-154. 1979.

[8] T. M. Cover and J. A. Thomas, Elements of Information Theory. Wiley, New York, 1991.

[9] W. Diffie and M. F. Hellman, New directions in cryptography, IEEE Transactions on Information Theon: vol. 22, pp. 644-654, Nov. 1976.

[10] L. M. Maurer. Secrel key agreement by public discussion from common informalion. IEEE Transactions on Information Theory, vol. 39, pp. 73.3-742, May 1993. 
[11] U. M. Maurer, The strong secret key rate of discrete random triples, In: Communications and Cryptography: Two Sides of One Tapestry (R. E. Blahut et al., eds.), Kluwer, Amsterdam, 1994.

[12] A. Rényi, On measures of entropy and information, In: Proc. 4th Berkeley Symposium on Mathematical and Statistical Probability, vol. I (Berkeley), pp. 547-561, University of California Press, Berkeley, CA, 1961.

[13] P. W. Shor, Algorithms for quantum computation: Discrete logarithm and factoring, In: Proc. 35th IEEE Symposium on Foundations of Computer Science (FOCS), pp. 124-134, 1994. 